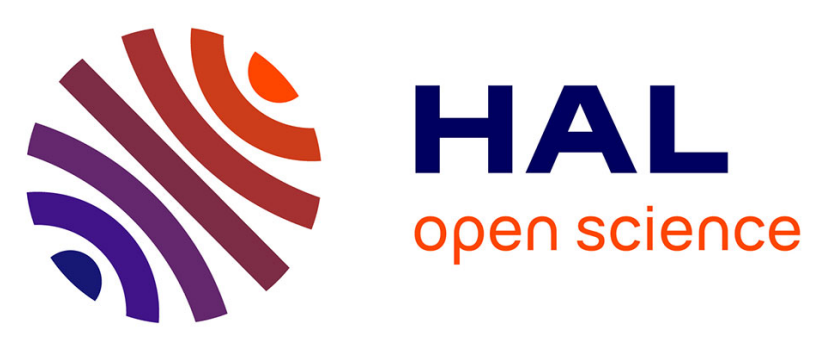

\title{
Exponential Stability With Decay Rate Estimation for Linear Difference Equations
}

Sérine Damak, Michaël Di Loreto, Sabine Mondié, Xavier Brun

\section{To cite this version:}

Sérine Damak, Michaël Di Loreto, Sabine Mondié, Xavier Brun. Exponential Stability With Decay Rate Estimation for Linear Difference Equations. IEEE Transactions on Automatic Control, 2016, 61 (1), pp.252-257. 10.1109/TAC.2015.2437519 . hal-01256040

\section{HAL Id: hal-01256040 https://hal.science/hal-01256040}

Submitted on 23 Apr 2019

HAL is a multi-disciplinary open access archive for the deposit and dissemination of scientific research documents, whether they are published or not. The documents may come from teaching and research institutions in France or abroad, or from public or private research centers.
L'archive ouverte pluridisciplinaire HAL, est destinée au dépôt et à la diffusion de documents scientifiques de niveau recherche, publiés ou non, émanant des établissements d'enseignement et de recherche français ou étrangers, des laboratoires publics ou privés. 


\title{
Exponential stability with decay rate estimation for linear difference equations
}

\author{
Sérine Damak, Michael Di Loreto, Sabine Mondié, Xavier Brun
}

\begin{abstract}
This technical note addresses the stability analysis of linear systems governed by continuous-time difference equations with multiple delays. We propose new sufficient conditions for $L_{2}$-exponential stability and exponential stability within the Lyapunov-Krasovskii framework. These conditions are delay-independent, and provide constructive exponential decay rate estimates of the system response. It is also shown that these conditions are less conservative than previous results in the literature.
\end{abstract}

Index Terms-Time-Delay Systems, Stability, Linear Systems

\section{INTRODUCTION}

Linear systems governed by continuous-time difference equations are dynamical systems with a state-space realization described by

$$
x(t)=\sum_{k=1}^{N} A_{k} x\left(t-r_{k}\right), t \geq 0,
$$

where $x(\cdot) \in \mathbb{R}^{n}$ is the instantaneous state, $r_{k}>0$ are the delays and $A_{k} \in \mathbb{R}^{n \times n}$, for $k=1, \ldots, N$. Difference equations in the form (1) appear in systems of conservation laws modeled by first order hyperbolic partial differential equations, where the delay operator describes some transport phenomenon with finite velocity [5], [3], in coupled differential-difference equations [9], [16], [15], neutral timedelay systems [10], [12], [7], [13], integral equations or difference equations with distributed delays [2], [11], [14], and in references therein, covering a wide class of applications, as electric transmission lines, road traffic, water channels or gas flow in pipeline networks.

Exponential stability analysis for systems in the form (1) is a crucial problem for transient response and steady-state analysis, closed-loop performances and stabilizing control synthesis. Exponential stability of (1) has been studied in [1], where it was proved that

$$
\sup _{\theta_{1}, \ldots, \theta_{N} \in[0,2 \pi]^{N}} \rho\left(\sum_{k=1}^{N} A_{k} \mathrm{e}^{j \theta_{k}}\right)<1,
$$

with $\rho(A)$ the spectral radius of $A$, is a necessary and sufficient condition for delay-independent exponential stability of system (1). Since (2) requires iterative optimization methods to be tested and is not constructive, in general, for state-feedback synthesis, some numerically tractable sufficient stability conditions were proposed. In [12] (see also the references therein), it was shown that the sufficient exponential stability (delay-independent) condition

$$
\sum_{k=1}^{N}\left\|A_{k}\right\|<1
$$

leads to constructive exponential estimates for the system response of (1). However, this condition appears to be highly conservative.

S. Damak, M. Di Loreto and X. Brun are with Université de Lyon, INSA-Lyon, Laboratoire Ampère, 20 Avenue Albert Einstein, Villeurbanne 69621, France (e-mail: serine.damak@insa-lyon.fr, michael.di-loreto@insalyon.fr, xavier.brun@insa-lyon.fr). S. Mondié is with Departamento de Control Automatico, Av. Instituto Politecnico Nacional 2508, Col. San Pedro Zacatenco, Del. G.A. Madero, 07360 Mexico D.F., Mexico (e-mail: smondie@ctrl.cinvestav.mx)
As a first step for conservatism reduction with respect to (3), an $L_{2}$ stability analysis was considered in [4], using a Lyapunov-Krasovskii approach. From some derived arguments on coupled differentialdifference equations, an equivalent stability condition for (1) was proposed in [8]. The approach in [4] was further investigated in [16] (see Theorem 4 and Corollary 5 therein) and in [6] for exponential estimates in $L_{2}$-exponential stability. For systems in the form (1), while Lyapunov-Krasovskii theory appears to be suitable for $L_{2}$ exponential stability, with concessions regarding the inherent conservatism, the fact that the solution of (1) has in general step discontinuities and its state-space realization (1) has only difference operators, makes the analysis of the solution behavior at any time more intricate than its analysis in $L_{2}$-norm.

The contributions of this paper are focused on Lyapunov-Krasovskii methods for stability of the system (1), and are twofold. First, in order to reduce the inherent conservatism introduced within the approach in [4], an extended Lyapunov-Krasovskii functional is defined and used to obtain a new $L_{2}$-exponential stability delay-independent condition, which appears to be less conservative than the results in [6]. Second, an exponential stability delay-independent condition is derived. Constructive exponential decay rate estimates are provided, and the conservatism reduction is discussed and compared with already existing results in both contributions.

The paper is organized as follows. Section II contains the required background and material for the content of this paper. Section III addresses $L_{2}$-exponential stability with constructive estimates for the decay rate of the system response. Exponential stability is analyzed in Section IV. A discussion on the conservatism for the proposed method is included in Section V.

Notations: The transpose of a matrix $P$ is denoted by $P^{T}$, while the smallest and the largest eigenvalues of a symmetric matrix $P$ are denoted by $\lambda_{\min }(P)$ and $\lambda_{\max }(P)$, respectively. The standard notation $P \prec Q(P \preceq Q)$ means that $Q-P$ is a symmetric positive definite matrix (semidefinite matrix, respectively). For a symmetric positive definite matrix $P, P^{1 / 2}$ stands for the (unique) symmetric positive definite square root matrix of $P$. Symmetric terms in symmetric matrices are denoted by $\star$. The notation $\operatorname{bd}\left(P_{k}\right)$ for $k=1, \ldots, N$ stands for the block-diagonal matrix

$$
\operatorname{bd}\left(P_{1}, \ldots, P_{N}\right)=\left[\begin{array}{ccc}
P_{1} & & \\
& \ddots & \\
& & P_{N}
\end{array}\right] .
$$

The matrices $I_{n}$ and $0_{n \times m}$ are the $n \times n$ identity matrix and the $n \times m$ zero matrix, respectively, with indices that may be dropped if there is no ambiguity. The set of natural numbers is denoted by $\mathbb{N}$, and if 0 is not included, the set will be denoted by $\mathbb{N}^{*}$. The space of piecewise right-continuous and bounded functions defined on $\left[-r_{N}, 0\right)$ is $\mathscr{P}\left(\left[-r_{N}, 0\right), \mathbb{R}^{n}\right)$. This space is equipped with the uniform norm $\|\varphi\|_{c}=\sup _{-r_{N} \leq \theta<0}\|\varphi(\theta)\|$ or with the $L_{2}$ norm $\|\varphi\|_{L_{2}}^{2}=\int_{-r_{N}}^{0}\|\varphi(\theta)\|^{2} \mathrm{~d} \theta$, where $\|\varphi(\theta)\|$ stands for the euclidean norm. We denote $x(t, \varphi)$ the solution at time $t$ of the system with the initial condition $\varphi$ and $x_{t}(\varphi)=\left\{x(t+\theta, \varphi) \mid \theta \in\left[-r_{N}, 0\right)\right\}$. 
The dependency with respect to $\varphi$ is dropped when it is clear from the context. $D^{+} v\left(x_{t}\right)$ stands for the Dini upper right-hand derivative of a functional $v\left(x_{t}\right)$.

\section{Preliminaries}

Consider the linear dynamical system governed by the continuoustime difference equations in the form

$$
x(t)=\sum_{k=1}^{N} A_{k} x\left(t-r_{k}\right), t \geq 0,
$$

where $A_{k} \in \mathbb{R}^{n \times n}$ for $k=1, \ldots, N, 0<r_{1}<\ldots<r_{N}$. For any piecewise right-continuous and bounded initial condition $\varphi \in$ $\mathscr{P}\left(\left[-r_{N}, 0\right), \mathbb{R}^{n}\right)$, there exists a unique piecewise right-continuous solution $x(t, \varphi)$ of (4), for all $t \geq 0$. This solution is called the system response of (4).

Let us recall the definitions of stability which are used throughout the paper.

\section{Definition 1:}

(i) System (4) is said to be $L_{2}$-exponentially stable if there exist $\mu>0$ and $\beta \geq 0$ such that, for any $\varphi \in \mathscr{P}\left(\left[-r_{N}, 0\right), \mathbb{R}^{n}\right)$,

$$
\left\|x_{t}(\varphi)\right\|_{L_{2}} \leq \beta\|\varphi\|_{c} \mathrm{e}^{-\mu t}, t \geq 0 .
$$

(ii) System (4) is said to be exponentially stable if there exist $\sigma>0$ and $\gamma \geq 0$ such that, for any $\varphi \in \mathscr{P}\left(\left[-r_{N}, 0\right), \mathbb{R}^{n}\right)$,

$$
\|x(t, \varphi)\| \leq \gamma\|\varphi\|_{c} \mathrm{e}^{-\sigma t}, t \geq 0 .
$$

In Definition 1, the positive constants $\mu$ and $\sigma$ are lower bounds for the exponential decay rates of the system response in $L_{2}$-norm and euclidean norm, respectively.

Let us denote by $\mathbf{A}_{c}$ the $n N \times n N$ companion matrix

$$
\mathbf{A}_{c}=\left[\begin{array}{cccc}
A_{1} & A_{2} & \cdots & A_{N} \\
I & & & \\
& \ddots & & \\
& & I & 0
\end{array}\right] .
$$

It was proved in [4] Corollary 5.4 that, if there exist $n \times n$ symmetric positive definite matrices $P_{k}$, for $k=1, \ldots, N$, such that

$$
-\mathbf{M}_{0}=\mathbf{A}_{c}^{T} \mathbf{b d}\left(P_{1}, \ldots, P_{N}\right) \mathbf{A}_{c}-\mathbf{b d}\left(P_{1}, \ldots, P_{N}\right)
$$

is a negative definite matrix, then the system (4) is $L_{2}$-exponentially stable, and also exponentially stable since (2) holds. Based on the same Lyapunov-Krasovskii functional, [6] proposed an estimate of the decay rate for $L_{2}$-exponential stability, that is there exists $\mu>0$ and $\beta(\mu) \geq 0$ such that

$$
-\mathbf{M}_{\mu}=-\mathbf{M}_{0}+\mathbf{b d}\left(\left(1-\mathrm{e}^{-2 \mu r_{k}}\right)\left(P_{k}-P_{k+1}\right)\right)
$$

is a negative semidefinite matrix (with $P_{N+1}=0$ by convention) and $(i)$ in Definition 1 holds. Under an additive assumption on contraction for $\mathbf{M}_{\mu}$ in (7), exponential estimates for convergence of the step discontinuities in the system response were characterized in [6] (see Theorem 4 therein). Actually, this condition, which appears also in Theorem 4 and Corollary 5 of [16], leads to exponential stability, as stated in the following result.

Theorem 2: Assume that there exist symmetric positive definite matrices $P_{k}$, for $k=1, \ldots, N$, and $\mu>0$, such that $\mathbf{M}_{\mu}$ in (7) is a positive semidefinite matrix. For this solution, if

$$
\alpha=\sum_{k=1}^{N} \mathrm{e}^{-2 \mu r_{k}} \lambda_{\max }\left(Q_{k}-Q_{k+1}\right)<1,
$$

where $Q_{k}=P_{1}^{-1 / 2} P_{k} P_{1}^{-1 / 2}$ for $k=1, \ldots, N$, then the system (4) is exponentially stable, and the following exponential estimate of the system response holds

$$
\|x(t, \varphi)\| \leq \gamma\|\varphi\|_{c} \mathrm{e}^{-\sigma t}, \quad \forall t \geq 0,
$$

with

$$
\gamma=\sqrt{\frac{\lambda_{\max }\left(P_{1}\right)}{\lambda_{\min }\left(P_{1}\right)}}, \quad \text { and } \quad \sigma=-\frac{\ln (\alpha)}{2 r_{N}} .
$$

Proof: Obvious from the proof of Theorem 4 in [6].

It is worthy of mention that the condition $-\mathbf{M}_{\mu} \preceq 0$ in Theorem 2 is delay-independent, in the sense that if it is satisfied for some delays $\left(r_{1}, \ldots, r_{N}\right)$, it is also satisfied for any delays $\left(r_{1}^{\prime}, \ldots, r_{N}^{\prime}\right)$. Its solution is however delay-dependent: for the matrices $P_{k}$ and $\mu$ that satisfy $-\mathbf{M}_{\mu} \preceq 0$, inequality (8) may be feasible or not depending on the delays. For $L_{2}$-exponential stability, we will need the following lemma [6].

Lemma 3: Assume that there exists a continuous functional $v$ : $\mathscr{P}\left(\left[-r_{N}, 0\right), \mathbb{R}^{n}\right) \rightarrow \mathbb{R}$ such that $t \mapsto v\left(x_{t}(\varphi)\right)$ is Dini upper righthand differentiable for $t \geq 0$ and such that

(a) $\beta_{1}\|\varphi\|_{L_{2}}^{2} \leq v(\varphi) \leq \beta_{2}\|\varphi\|_{c}^{2}$, for some $\beta_{1}>0, \beta_{2}>0$,

(b) $\forall t \geq 0, D^{+} v\left(x_{t}(\varphi)\right)+2 \mu v\left(x_{t}(\varphi)\right) \leq 0$, for some $\mu>0$.

Then the system (4) is $L_{2}$-exponentially stable, that is

$$
\left\|x_{t}(\varphi)\right\|_{L_{2}} \leq \sqrt{\frac{\beta_{2}}{\beta_{1}}}\|\varphi\|_{c} \mathrm{e}^{-\mu t}, t \geq 0
$$

\section{III. $L_{2}$-EXPONENTIAL STABILITY}

The major source of conservatism of the results presented in the previous section is related to the considered Lyapunov-Krasovskii functional, corresponding to (see [4] and [6])

$$
\nu_{\mu}\left(x_{t}(\varphi)\right)=\sum_{k=1}^{N} \int_{t-r_{k}}^{t} \mathrm{e}^{-2 \mu(t-\theta)} x(\theta)^{T}\left(P_{k}-P_{k+1}\right) x(\theta) \mathrm{d} \theta,
$$

with $0 \prec P_{k}$, for $k=1, \ldots, N$ and $\mu>0$ the solutions of (7) in Theorem 2. In order to achieve less conservative stability conditions, the block-diagonal structure in (7) must be relaxed by increasing the number of decision variables in Theorem 2, which is realized by the introduction in the Lyapunov-Krasovskii functional (10) of delayed cross-weighted terms.

For this, let $S_{N}$ a $n \times n$ symmetric positive definite matrix, $n \times n$ symmetric positive semidefinite matrices $S_{i}$, for $i=1, \ldots, N-1$, $2 n \times 2 n$ symmetric positive semidefinite matrices $\mathbf{P}_{k}$ and $\mathbf{Q}_{k}$, for $k=2, \ldots, N$,

$$
\mathbf{P}_{k}=\left[\begin{array}{cc}
\mathbf{P}_{11}^{(k)} & \mathbf{P}_{12}^{(k)} \\
\star & \mathbf{P}_{22}^{(k)}
\end{array}\right], \mathbf{Q}_{k}=\left[\begin{array}{cc}
\mathbf{Q}_{11}^{(k)} & \mathbf{Q}_{12}^{(k)} \\
\star & \mathbf{Q}_{22}^{(k)}
\end{array}\right]
$$

with respective blocks of size $n \times n$, and denote the positive constants $\kappa_{k}=r_{k}-r_{k-1}$, for $k=2, \ldots, N$.

With these notations, consider the Lyapunov-Krasovskii functional

$$
v_{\mu}\left(x_{t}\right)=\sum_{i=1}^{N} v_{i}^{\mu}\left(x_{t}\right)+\sum_{k=2}^{N}\left(v_{\mathbf{P}, k}^{\mu}\left(x_{t}\right)+v_{\mathbf{Q}, k}^{\mu}\left(x_{t}\right)\right)
$$

where

$$
\begin{gathered}
v_{i}^{\mu}\left(x_{t}\right)=\int_{t-r_{i}}^{t} \mathrm{e}^{-2 \mu(t-\theta)} x(\theta)^{T} S_{i} x(\theta) \mathrm{d} \theta, \\
v_{\mathbf{P}, k}^{\mu}\left(x_{t}\right)=\int_{t-\kappa_{k}}^{t} \mathrm{e}^{-2 \mu(t-\theta)} \zeta_{k}(\theta)^{T} \mathbf{P}_{k} \zeta_{k}(\theta) \mathrm{d} \theta, \\
v_{\mathbf{Q}, k}^{\mu}\left(x_{t}\right)=\int_{t-r_{k}}^{t-\kappa_{k}} \mathrm{e}^{-2 \mu(t-\theta)} \xi_{k}(\theta)^{T} \mathbf{Q}_{k} \xi_{k}(\theta) \mathrm{d} \theta,
\end{gathered}
$$


$\zeta_{k}(\theta)=\left[\begin{array}{c}x(\theta) \\ x\left(\theta-r_{k-1}\right)\end{array}\right]$ and $\xi_{k}(\theta)=\left[\begin{array}{c}x\left(\theta+\kappa_{k}\right) \\ x(\theta)\end{array}\right]$.

The Lyapunov-Krasovskii functional introduced in (12) leads to the following $L_{2}$-exponential stability result.

Theorem 4: Assume that there exist a $n \times n$ symmetric positive definite matrix $S_{N}, n \times n$ symmetric positive semidefinite matrices $S_{i}$, for $i=1, \ldots, N-1,2 n \times 2 n$ symmetric positive semidefinite matrices $\mathbf{P}_{k}$ and $\mathbf{Q}_{k}$, for $k=2, \ldots, N$, and $\mu>0$ such that

$$
-\boldsymbol{\Lambda}_{\mu}=\left[\begin{array}{ll}
\boldsymbol{\Psi}_{\mu} & \boldsymbol{\Upsilon}_{\mu} \\
\boldsymbol{\Upsilon}_{\mu}^{T} & \mathbf{\Phi}_{\mu}
\end{array}\right]
$$

is a $(2 N-1) n \times(2 N-1) n$ negative semidefinite matrix, where

$$
\begin{aligned}
\mathbf{\Psi}_{\mu} & =\mathbf{A}_{c}^{T} \mathbf{W} \mathbf{A}_{c}-\mathbf{W}_{P}-\mathbf{W}_{Q} \\
\boldsymbol{\Phi}_{\mu} & =\mathbf{b d}\left(\mathrm{e}^{-2 \mu \kappa_{k}}\left(\mathbf{Q}_{22}^{(k)}-\mathbf{P}_{11}^{(k)}\right)\right) \\
\mathbf{\Upsilon}_{\mu}=\mathbf{A}_{c}^{T} & {\left[\begin{array}{ccc}
\mathrm{e}^{-2 \mu \kappa_{2}} \mathbf{Q}_{12}^{(2)} & \cdots & \mathrm{e}^{-2 \mu \kappa_{N}} \mathbf{Q}_{12}^{(N)} \\
0_{(N-1) n \times n} & \cdots & 0_{(N-1) n \times n}
\end{array}\right] } \\
- & {\left[\begin{array}{c}
0_{n \times(N-1) n} \\
\mathbf{b d}\left(\mathrm{e}^{-2 \mu \kappa_{k}} \mathbf{P}_{12}^{(k)^{T}}\right)
\end{array}\right] }
\end{aligned}
$$

and $\mathbf{W}, \mathbf{W}_{P}, \mathbf{W}_{Q}$ are defined in (23)-(25), respectively. Then, the system (4) is $L_{2}$-exponentially stable. Furthermore, the following exponential estimate holds

$$
\left\|x_{t}(\varphi)\right\|_{L_{2}} \leq \sqrt{\frac{\beta_{2}}{\beta_{1}}}\|\varphi\|_{c} \mathrm{e}^{-\mu t}, t \geq 0
$$

with

$$
\begin{aligned}
\beta_{1}= & 2 \min _{k=2, \ldots, N}\left\{\mathrm{e}^{-2 \mu \kappa_{k}} \lambda_{\min }\left(\mathbf{P}_{k}\right), \mathrm{e}^{-2 \mu r_{k}} \lambda_{\min }\left(\mathbf{Q}_{k}\right)\right\} \\
& +\mathrm{e}^{-2 \mu r_{N}} \lambda_{\min }\left(S_{N}\right), \\
\beta_{2}= & 2 \sum_{k=2}^{N}\left(\kappa_{k} \lambda_{\max }\left(\mathbf{P}_{k}\right)+r_{k-1} \mathrm{e}^{-2 \mu \kappa_{k}} \lambda_{\max }\left(\mathbf{Q}_{k}\right)\right) \\
& +\sum_{k=1}^{N} r_{k} \lambda_{\max }\left(S_{k}\right) .
\end{aligned}
$$

Proof: The functional $v_{\mu}\left(x_{t}(\varphi)\right)$ in (12) is well-defined over $\mathscr{P}\left(\left[-r_{N}, 0\right), \mathbb{R}^{n}\right)$, continuous with respect to $t$, and Dini upper righthand differentiable for any $t \geq 0$ along the trajectory of (4). Simple computations lead to

$$
\beta_{1}\|\varphi\|_{L_{2}}^{2} \leq v_{\mu}(\varphi) \leq \beta_{2}\|\varphi\|_{c}^{2}
$$

where $\beta_{1}>0$ and $\beta_{2}>0$ are defined in (21) and (22), respectively. For $i=1, \ldots, N$, the Dini upper right-hand derivative of $v_{i}^{\mu}\left(x_{t}(\varphi)\right)$ in (13) along the trajectory of (4) is

$$
\begin{aligned}
D^{+} v_{i}^{\mu}\left(x_{t}\right)= & -2 \mu v_{i}^{\mu}\left(x_{t}\right)+x(t)^{T} S_{i} x(t) \\
& -\mathrm{e}^{-2 \mu r_{i}} x\left(t-r_{i}\right)^{T} S_{i} x\left(t-r_{i}\right) .
\end{aligned}
$$

Similarly, concerning (14) and (15), we have, for any $k=2, \ldots, N$,

$$
\begin{aligned}
D^{+} v_{\mathbf{P}, k}^{\mu}\left(x_{t}\right)= & -2 \mu v_{\mathbf{P}, k}^{\mu}\left(x_{t}\right)+\zeta_{k}(t)^{T} \mathbf{P}_{k} \zeta_{k}(t) \\
& -\mathrm{e}^{-2 \mu \kappa_{k}} \zeta_{k}\left(t-\kappa_{k}\right)^{T} \mathbf{P}_{k} \zeta_{k}\left(t-\kappa_{k}\right)
\end{aligned}
$$

and

$$
\begin{aligned}
D^{+} v_{\mathbf{Q}, k}^{\mu}\left(x_{t}\right)= & -2 \mu v_{\mathbf{Q}, k}^{\mu}\left(x_{t}\right) \\
& +\mathrm{e}^{-2 \mu \kappa_{k}} \xi_{k}\left(t-\kappa_{k}\right)^{T} \mathbf{Q}_{k} \xi_{k}\left(t-\kappa_{k}\right) \\
& -\mathrm{e}^{-2 \mu r_{k}} \xi_{k}\left(t-r_{k}\right)^{T} \mathbf{Q}_{k} \xi_{k}\left(t-r_{k}\right) .
\end{aligned}
$$

Substitute (4) into these expressions, and using the decomposition blocks (11) in (12), we arrive at the Dini upper right-hand derivative of $v_{\mu}\left(x_{t}(\varphi)\right)$ along the trajectory of (4), that is

$$
D^{+} v_{\mu}\left(x_{t}(\varphi)\right)=-2 \mu v_{\mu}\left(x_{t}(\varphi)\right)-\chi(t)^{T} \boldsymbol{\Lambda}_{\mu} \chi(t),
$$

where $0 \preceq \Lambda_{\mu}$ is defined in (16) and $\chi(t)=\left[\begin{array}{ll}\chi_{1}(t)^{T} & \chi_{2}(t)^{T}\end{array}\right]^{T}$,

$$
\chi_{1}(t)=\left[\begin{array}{c}
x\left(t-r_{1}\right) \\
\vdots \\
x\left(t-r_{N}\right)
\end{array}\right], \chi_{2}(t)=\left[\begin{array}{c}
x\left(t-\kappa_{2}\right) \\
\vdots \\
x\left(t-\kappa_{N}\right)
\end{array}\right] .
$$

It follows from (27) that $D^{+} v_{\mu}\left(x_{t}(\varphi)\right)+2 \mu v_{\mu}\left(x_{t}(\varphi)\right) \leq 0$, for any $t \geq 0$. Lemma 3 applies and the proof is completed.

Remark 5: Note that with respect to [4], if there exist $P_{k} \succ 0$, for $k=1, \ldots, N$, and $\mu>0$ such that $-\mathbf{M}_{\mu} \preceq 0$ in (7), Theorem 2 in [6] leads to $L_{2}$-exponential stability, with the Lyapunov-Krasovskii functional (10). This implies of course that Theorem 4 is fulfilled, with $\mathbf{P}_{k}=\mathbf{Q}_{k}=0$ for $k=2, \ldots, N$, and $S_{k}=P_{k}-P_{k+1}$ for $k=1, \ldots, N$ (with $P_{N+1}=0$ ). The matrices $P_{k}$ satisfy, using the block-diagonal terms in (7), $P_{N} \preceq P_{N-1} \preceq \cdots \preceq P_{1}$. This implies that $S_{i}$ are positive semidefinite matrices, and $S_{N}=P_{N}$ is positive definite. The converse is false, as illustrated and commented in Section V.

The condition given in Theorem 4 is delay-independent. It is equivalent to the existence of $S_{N} \succ 0, S_{i} \succeq 0$ for $i=1, \ldots, N-1$, $\mathbf{P}_{k} \succeq 0$ and $\mathbf{Q}_{k} \succeq 0$ for $k=2, \ldots, N$, such that $-\boldsymbol{\Lambda}_{0} \prec 0$. This fact is outlined in the following result, where the necessary and sufficient delay-independent condition (2) is implied.

Theorem 6: Assume that there exist a $n \times n$ symmetric positive definite matrix $S_{N}, n \times n$ symmetric positive semidefinite matrices $S_{i}$, for $i=1, \ldots, N-1,2 n \times 2 n$ symmetric positive semidefinite matrices $\mathbf{P}_{k}$ and $\mathbf{Q}_{k}$, for $k=2, \ldots, N$, and $\mu>0$ such that $\boldsymbol{\Lambda}_{\mu}$ in (16) is a $(2 N-1) n \times(2 N-1) n$ positive semidefinite matrix. Then

$$
\sup _{\theta_{1}, \ldots, \theta_{N} \in[0,2 \pi]^{N}} \rho\left(\sum_{k=1}^{N} A_{k} \mathrm{e}^{j \theta_{k}}\right)<1 .
$$

Proof: Under the specified assumptions and for any $\theta_{i} \in[0,2 \pi]$, $i=1, \ldots, N$, we compute the quadratic form $-u^{*} \boldsymbol{\Lambda}_{\mu} u \leq 0$ with

$$
u=\left[\begin{array}{c}
\mathrm{e}^{j \theta_{1}} I_{n} \\
\vdots \\
\mathrm{e}^{j \theta_{N}} I_{n} \\
\sum_{i=1}^{N} A_{i} \mathrm{e}^{j\left(\theta_{i}-\theta_{1}+\theta_{2}\right)} \\
\vdots \\
\sum_{i=1}^{N} A_{i} \mathrm{e}^{j\left(\theta_{i}-\theta_{N-1}+\theta_{N}\right)}
\end{array}\right] .
$$

We obtain

$$
-u^{*} \boldsymbol{\Lambda}_{\mu} u=\left(\sum_{k=1}^{N} A_{k} \mathrm{e}^{j \theta_{k}}\right)^{*} \mathbf{Y}\left(\sum_{k=1}^{N} A_{k} \mathrm{e}^{j \theta_{k}}\right)-\tilde{\mathbf{Y}}+\mathbf{S}
$$

where

$$
\begin{aligned}
\mathbf{Y} & =\sum_{l=1}^{N} S_{l}+\sum_{k=2}^{N} \mathrm{e}^{-2 \mu \kappa_{k}} \mathbf{H}_{k}, \\
\widetilde{\mathbf{Y}} & =\sum_{l=1}^{N} \mathrm{e}^{-2 \mu r_{l}} S_{l}+\sum_{k=2}^{N} \mathrm{e}^{-2 \mu r_{k}} \mathbf{H}_{k}, \\
\mathbf{S} & =\sum_{k=2}^{N}\left(1-\mathrm{e}^{-2 \mu \kappa_{k}}\right) \mathbf{R}_{k},
\end{aligned}
$$




$$
\begin{aligned}
& \mathbf{W}=\left[\begin{array}{ccccc}
\Xi & \mathbf{P}_{12}^{(2)} & \mathbf{P}_{12}^{(3)} & \cdots & \mathbf{P}_{12}^{(N)} \\
\star & \mathbf{P}_{22}^{(2)} & & & \\
\star & & \mathbf{P}_{22}^{(3)} & & \\
\vdots & & & \ddots & \\
\star & & & & \mathbf{P}_{22}^{(N)}
\end{array}\right], \Xi=\sum_{i=1}^{N} S_{i}+\sum_{k=2}^{N}\left(\mathbf{P}_{11}^{(k)}+\mathrm{e}^{-2 \mu \kappa_{k}} \mathbf{Q}_{11}^{(k)}\right) \\
& \mathbf{W}_{P}=\operatorname{bd}\left(\mathrm{e}^{-2 \mu r_{1}} S_{1}, \mathrm{e}^{-2 \mu \kappa_{2}} \mathbf{P}_{22}^{(2)}+\mathrm{e}^{-2 \mu r_{2}} S_{2}, \ldots, \mathrm{e}^{-2 \mu \kappa_{N}} \mathbf{P}_{22}^{(N)}+\mathrm{e}^{-2 \mu r_{N}} S_{N}\right)
\end{aligned}
$$

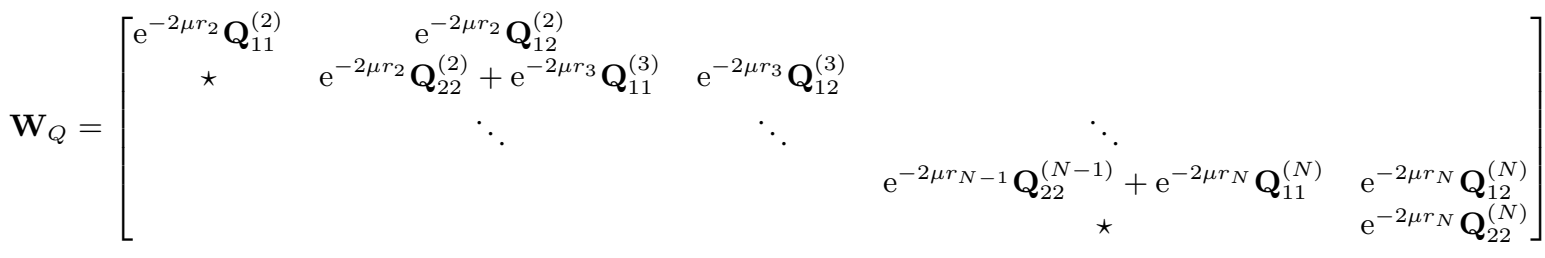

with

$$
\mathbf{H}_{k}=\left[\begin{array}{c}
I_{n} \\
\mathrm{e}^{j\left(\theta_{k}-\theta_{k-1}\right)} I_{n}
\end{array}\right]^{*} \mathbf{Q}_{k}\left[\begin{array}{c}
I_{n} \\
\mathrm{e}^{j\left(\theta_{k}-\theta_{k-1}\right)} I_{n}
\end{array}\right]
$$

and

$$
\mathbf{R}_{k}=\left[\begin{array}{c}
\sum_{i=1}^{N} A_{i} \mathrm{e}^{j \theta_{i}} \mathrm{e}^{-j \theta_{k-1}} \\
I_{n}
\end{array}\right]^{*} \mathbf{P}_{k}\left[\begin{array}{c}
\sum_{i=1}^{N} A_{i} \mathrm{e}^{j \theta_{i}} \mathrm{e}^{-j \theta_{k-1}} \\
I_{n}
\end{array}\right] .
$$

Note that $\mathbf{H}_{k}$ and $\mathbf{R}_{k}$ defined in (34) and (35), respectively, are hermitian positive semidefinite matrices, for any $k=2, \ldots, N$ and for any $\theta_{i} \in[0,2 \pi], i=1, \ldots, N$. Since $\mathbf{S}$ defined in (33) is a positive semidefinite matrix, the negative term (30) implies that

$$
\left(\sum_{k=1}^{N} A_{k} \mathrm{e}^{j \theta_{k}}\right)^{*} \mathbf{Y}\left(\sum_{k=1}^{N} A_{k} \mathrm{e}^{j \theta_{k}}\right)-\widetilde{\mathbf{Y}} \preceq 0 .
$$

Furthermore, using (31), (32) and the fact that $S_{N}$ is positive definite, we see that $\mathbf{Y}$ is positive definite and $\widetilde{\mathbf{Y}} \prec \mathbf{Y}$. Applying this last inequality in (36) leads to

$$
\left(\sum_{k=1}^{N} A_{k} \mathrm{e}^{j \theta_{k}}\right)^{*} \mathbf{Y}\left(\sum_{k=1}^{N} A_{k} \mathrm{e}^{j \theta_{k}}\right)-\mathbf{Y} \prec 0 .
$$

It follows that $\rho\left(\sum_{k=1}^{N} A_{k} \mathrm{e}^{j \theta_{k}}\right)<1$, for any $\theta_{k} \in[0,2 \pi]$, $k=1, \ldots, N$. This concludes the proof.

\section{EXPONENTIAL STABILITY}

According to Theorem 3.5 with inequality (3.22) and Theorem 6.1 p. 286 in [10], Theorem 6 leads to delay-independent exponential stability, with however non constructive estimates. In order to evaluate the performance of the system (4) through its exponential decay rate, we assume that the conditions of Theorem 4 are fulfilled. Estimates for exponential stability are given in the following result.

Lemma 7: Assume that there exist $\beta \geq 0$ and $\mu>0$ such that, for any $\varphi \in \mathscr{P}\left(\left[-r_{N}, 0\right), \mathbb{R}^{n}\right)$ and for any $t \geq 0$,

$$
\left\|x_{t}(\varphi)\right\|_{L_{2}} \leq \beta\|\varphi\|_{c} \mathrm{e}^{-\mu t}
$$

Then we have

$$
\|x(t, \varphi)\| \leq \frac{\beta}{\sqrt{r_{N}}}\|\varphi\|_{c} \mathrm{e}^{-\mu t}, \text { a.e. } t \geq 0 .
$$

Proof: Let us first define the function $\phi(\vartheta)=\int_{-r_{N}}^{\vartheta}\|x(\theta)\|^{2} \mathrm{~d} \theta$ with $\vartheta \geq-r_{N}$ which is continuous, non negative, non decreasing and bounded, since from (38), $\phi(\infty) \leq \beta^{2}\|\varphi\|_{c}^{2} \frac{1}{1-\mathrm{e}^{-2 \mu r} N}$. This function allows us to rewrite the inequality (38) as

$$
\phi(t)-\phi\left(t-r_{N}\right) \leq \beta^{2}\|\varphi\|_{c}^{2} \mathrm{e}^{-2 \mu t}, t \geq 0 .
$$

For an arbitrary $\varsigma \in \mathbb{N}^{*}$, multiplying (40) by $r_{N}^{-1}$ and introducing intermediate values of $\phi(\cdot)$, we obtain for any $t \geq 0$,

$$
\frac{1}{\varsigma} \sum_{i=0}^{\varsigma-1} \omega_{\varsigma}\left(t-i \frac{r_{N}}{\varsigma}\right) \leq \frac{\beta^{2}}{r_{N}}\|\varphi\|_{c}^{2} \mathrm{e}^{-2 \mu t}
$$

where $\omega_{\varsigma}(t)=\frac{\phi(t)-\phi\left(t-\frac{r_{N}}{\varsigma}\right)}{\frac{r_{N}}{\varsigma}}$. This function is also continuous, non negative and bounded for any $t \geq 0$. For any $i=0, \ldots, \varsigma-1$,

$$
\begin{aligned}
\omega_{\varsigma}\left(t-i \frac{r_{N}}{\varsigma}\right) & \geq \min _{i=0, \ldots, \varsigma-1} \omega_{\varsigma}\left(t-i \frac{r_{N}}{\varsigma}\right) \\
& \geq \min _{0 \leq \chi \leq 1} \omega_{\varsigma}\left(t-r_{N} \chi\right)=\omega_{\varsigma}\left(t-r_{N} \chi_{\varsigma}^{*}\right),
\end{aligned}
$$

such that $\chi_{\varsigma}^{*} \in[0,1]$, for all $\varsigma \in \mathbb{N}^{*}$. Using this inequality into (41),

$$
\omega_{\varsigma}\left(t-r_{N} \chi_{\varsigma}^{*}\right) \leq \frac{\beta^{2}}{r_{N}}\|\varphi\|_{c}^{2} \mathrm{e}^{-2 \mu t}, t \geq 0
$$

Since the function $\omega_{\varsigma}(\cdot)$ is continuous, we obtain, taking the limit sup when $\varsigma \rightarrow \infty$ in (42), that

$$
\begin{aligned}
\limsup _{\varsigma \rightarrow \infty} \omega_{\varsigma}\left(t-r_{N} \chi_{\varsigma}^{*}\right) & =\limsup _{\varsigma \rightarrow \infty} \omega_{\varsigma}\left(t-r_{N} \chi_{\infty}^{*}\right) \\
& =D^{+} \phi\left(t-r_{N} \chi_{\infty}^{*}\right) \leq \frac{\beta^{2}}{r_{N}}\|\varphi\|_{c}^{2} \mathrm{e}^{-2 \mu t}
\end{aligned}
$$

holds for any $t \geq 0$. Since $D^{+} \phi(t)=\|x(t, \varphi)\|^{2}$ a.e. and $\mathrm{e}^{-2 \mu r_{N} \chi_{\infty}^{*}} \leq 1$, it follows that

$$
\|x(t, \varphi)\|^{2} \leq \frac{\beta^{2}}{r_{N}}\|\varphi\|_{c}^{2} \mathrm{e}^{-2 \mu t} \text {, a.e. } t \geq 0
$$

that is (39) is satisfied.

The main advantage of Lemma 7 is that, unlike in Theorem 2, no additional assumption is required to get constructive estimates for exponential stability from estimates for $L_{2}$-exponential stability. Note that (39) holds almost everywhere for $t \geq 0$. While the step discontinuities in the system response are ensured, from Theorem 6, to converge exponentially to zero, estimates for such a convergence are not obtained in Lemma 7. A direct application of Lemma 7 to Theorem 4 leads to the following result on exponential stability.

Theorem 8: Assume that there exist a $n \times n$ symmetric positive definite matrix $S_{N}, n \times n$ symmetric positive semidefinite matrices $S_{i}$, for $i=1, \ldots, N-1,2 n \times 2 n$ symmetric positive semidefinite matrices $\mathbf{P}_{k}$ and $\mathbf{Q}_{k}$, for $k=2, \ldots, N$, and $\mu>0$ such that $\boldsymbol{\Lambda}_{\mu}$ defined in (16) is a positive semidefinite matrix. Then, the system (4) is exponentially stable, and its system response satisfies

$$
\|x(t, \varphi)\| \leq \sqrt{\frac{\beta_{2}}{r_{N} \beta_{1}}}\|\varphi\|_{c} \mathrm{e}^{-\mu t}, \text { a.e. } t \geq 0
$$


where $\beta_{1}$ and $\beta_{2}$ are given in (21) and (22), respectively.

Proof: Obvious from Theorem 4 and Lemma 7.

\section{CONSERVATISM ANALYSIS AND DISCUSSION}

We prove first that Theorem 4 is less conservative than Corollary 5.4 in [4] or Theorem 2 in [6].

As outlined in Remark 5, it is clear that if Corollary 5.4 in [4] is fulfilled, then Theorem 4 holds, leading to a delay-independent exponential stability. However, the converse is false. Indeed, let us consider the system

$$
x(t)=A_{1} x\left(t-r_{1}\right)+A_{2} x\left(t-r_{2}\right),
$$

where

$$
A_{1}=\left[\begin{array}{cc}
\frac{1}{2} & 0 \\
0 & -\frac{1}{2}
\end{array}\right], A_{2}=\left[\begin{array}{cc}
0 & \frac{1}{2} \\
-\frac{1}{2} & 0
\end{array}\right] .
$$

It was proved in [4] that for this system, the condition $-\mathbf{M}_{0} \prec 0$ in (6) has no solution, or equivalently $-\mathbf{M}_{\mu} \prec 0$ in (7) is not feasible. However, a solution of Theorem 4 exists. For $r_{1}=1$ and $r_{2}=\sqrt{2}$, we found $\mu=0.2842,\left\|x_{t}(\varphi)\right\|_{L_{2}} \leq 3.9989\|\varphi\|_{c} \mathrm{e}^{-0.2842 t}$, for $t \geq 0$, and Theorem 8 leads to $\|x(t, \varphi)\| \leq 3.3627\|\varphi\|_{c} \mathrm{e}^{-0.2842 t}$. Note that the computational cost of the proposed method requires $\frac{n(n+1)}{2}(5 N-4)+2 n^{2}(N-1)$ decision variables and $3 N-1$ constraint inequalities comparing to $\frac{n(n+1)}{2} N$ decision variables and $N+1$ constraint inequalities for the condition (6) in [4]. On the other hand, this increase allows us to reduce the conservatism.

Second, it is of interest to mention the conservatism improvement of our method with respect to the norm condition (3). For this, we just prove that (3) implies that Theorem 2 is satisfied.

Assume that (3) holds. By assumption, it is straightforward to see that

$$
\left\|A_{e}\right\|=\left\|\left[\begin{array}{ccc}
A_{1} & \cdots & A_{N} \\
0_{(N-1) n \times n} & \cdots & 0_{(N-1) n \times n}
\end{array}\right]\right\|<1 .
$$

Let $\lambda_{1}>0$ be any positive constant. The spectral theorem for symmetric matrices and (45) imply that $A_{e}^{T} \lambda_{1} A_{e} \prec \lambda_{1} \cdot I_{n N}$. For $P_{1}=\lambda_{1} \cdot I_{n}$, this last inequality rewrites as

$$
A_{e}^{T} \mathbf{b d}\left(P_{1}\right) A_{e}-\mathbf{b d}\left(P_{1}\right) \prec 0 .
$$

Since the previous inequality is strict, there exist sufficiently small positive constants $\varepsilon_{1}, \ldots, \varepsilon_{N}$ satisfying $\varepsilon_{N}<\ldots<\varepsilon_{1}, \frac{N-2}{N-1} \varepsilon_{1}<$ $\varepsilon_{N}$ and $A_{e}^{T} \mathbf{b d}\left(P_{1}\right) A_{e}-\mathbf{b d}\left(P_{1}-\varepsilon_{k} \cdot I_{n}\right) \preceq 0$. Let us define $\mu>0$ such that $\mu<-\frac{1}{2 r_{N}} \ln \left((N-1)\left(1-\frac{\varepsilon_{N}}{\varepsilon_{1}}\right)\right)$, and the matrices $P_{k}=$ $\lambda_{k} \cdot I_{n}$, for $k=1, \ldots, N$, obtained iteratively by

$$
\begin{aligned}
\lambda_{1} & =\frac{\sum_{k=1}^{N} \mathrm{e}^{2 \mu r_{k}} \varepsilon_{k}}{\sum_{k=1}^{N} \mathrm{e}^{2 \mu r_{k}}-1}, \\
\lambda_{k}-\lambda_{k+1} & =\mathrm{e}^{2 \mu r_{k}}\left(\lambda_{1}-\varepsilon_{k}\right) .
\end{aligned}
$$

The matrices $P_{k}$ satisfy $P_{k}-P_{k+1}=\mathrm{e}^{2 \mu r_{k}}\left(P_{1}-\varepsilon_{k} \cdot I_{n}\right)$, so that $P_{k+1} \prec P_{k}$, for $k=1, \ldots, N$. Since $P_{N}=\mathrm{e}^{2 \mu r_{N}}\left(P_{1}-\varepsilon_{N} \cdot I_{n}\right) \succ 0$, all the matrices $P_{k}$ are positive definite and diagonal. It follows that the matrices $P_{k} \succ 0, k=1, \ldots, N$, and the positive constant $\mu$, satisfy $\mathbf{M}_{\mu} \succeq 0$. Furthermore, as these matrices are in the form $P_{k}=\lambda_{k} \cdot I_{n},(8)$ is satisfied since $\alpha<\mathrm{e}^{-2 \mu r_{1}}$. Hence, Theorem 2 is fulfilled with $\gamma=1$ in (9), and consequently Theorem 4 holds. The converse of this assertion is false, as shown in the example below.

Example 9: Consider the difference equation (43) with $r_{1}=1$, $r_{2}=\pi$ and

$$
A_{1}=\left[\begin{array}{cc}
-0.4 & -0.3 \\
0.1 & 0.15
\end{array}\right], \quad A_{2}=\left[\begin{array}{cc}
0.1 & 0.25 \\
-0.9 & -0.1
\end{array}\right] \text {. }
$$

These matrices satisfy $\sum_{k=1}^{N}\left\|A_{k}\right\|=1.4435$, therefore the exponential stability of (43) cannot be analyzed using the conditions in [12].
However, this system is exponentially stable. Indeed, Theorem 2 leads to $\mu=0.1084, \alpha=0.9084$ in (8), and $\|x(t, \varphi)\| \leq$ $1.8245\|\varphi\|_{c} \mathrm{e}^{-0.0153 t}$, for any $t \geq 0$. On the other hand, Theorem 8 leads to the estimate

$$
\|x(t, \varphi)\| \leq 3.6514\|\varphi\|_{c} \mathrm{e}^{-0.1231 t}, t \geq 0 .
$$

Theorem 4 gives only sufficient conditions for the exponential stability of (4), which are less conservative than those proposed previously in the literature. When the delays are arbitrary but commensurate, that is $r_{k}=\xi_{k} r$ for $\xi_{k} \in \mathbb{N}^{*}$ and some $r>0$, the conditions in Theorem 4 appear to be necessary and sufficient for exponential stability. Indeed, if (4) is exponentially stable for any commensurate delays, this implies in particular that $\rho\left(\mathbf{A}_{c}\right)<1$, or equivalently that there exist $P \succ 0$ and $\mu>0$ such that

$$
\mathbf{A}_{c}^{T} P \mathbf{A}_{c}-\mathrm{e}^{-2 \mu r} P \preceq 0,
$$

which is precisely the condition $-\mathbf{M}_{\mu} \preceq 0$ given in (7) for the one-delay case. This in turn implies that Theorem 4 holds. The converse is obvious from Theorem 8 since delay-independent stability is characterized therein.

\section{CONCLUDING REMARKS}

New exponential stability sufficient conditions providing the decay estimate of the response of linear difference equations in continuous time are presented. The a priori norm condition which is required in similar results in the literature is lifted, thus allowing the study of a wider class of systems.

\section{ACKNOWLEDGMENT}

The authors acknowledge the financial supports of ANR under research grant AIDS, and of Conacyt, Mexico, under grant 180725.

\section{REFERENCES}

[1] Avellar C. E. and Hale J. K., "On the zeros of exponentials polynomials," Journal of Mathematical Analysis and Applications, 73, 434-452, 1980.

[2] Bellman, R., Cooke, K.L., Differential-Difference Equations, Academic Press: New-York, 1963.

[3] Bressan, A., Hyperbolic systems of conservation laws. The one dimensional Cauchy problem. Oxford University Press, Oxford: U-K, 2000.

[4] Carvalho L.A.V., "On quadratic Liapunov functionals for linear difference equations," Linear Alg. and its Appl., 240, 41-64, 1996.

[5] Coron J.M. and Bastin G. and D'Andréa-Novel B., "Dissipative boundary conditions for one-dimensional nonlinear hyperbolic systems," SIAM J. Control Optim., 47(3), 1460-1498, 2008.

[6] Damak S. and Di Loreto M. and Lombardi W. and Andrieu V., "Stability analysis for a class of linear systems governed by continuous-time difference equations," Automatica, to appear, 2014

[7] Fridman E., New Lyapunov-Krasovskii functionals for stability of linear retarded and neutral type systems. Syst. Contr. Lett., 43, 309-319, 2001.

[8] Fridman E., Stability of linear descriptor systems with delay : a Lyapunovbased approach. J. Math. Anal. Appl., 273, 24-44, 2002.

[9] Gu K., "Stability problem of systems with multiple delay channels," Automatica, 46, 743-751, 2010.

[10] Hale J. K. and Verduyn Lunel S. M., Introduction to functional differential equations, Springer-Verlag: New-York, 1993.

[11] Jerri, A.J., Introduction to integral equations with applications. 2nd Ed., Wiley: New-York, 1999.

[12] Kharitonov V. and Collado J. and Mondié S., Exponential estimates for neutral time delay systems with multiple delays, J. Robust Nonlinear Control, 16, 71-84, 2006.

[13] Kharitonov V., Time delay systems: Lyapunov functionals and matrices, Birkhäuser, 2013.

[14] Melchor-Aguilar D., Exponential stability of linear continuous time difference systems with multiple delays. Syst. Contr. Lett., 62, 811-818, 2013.

[15] Pepe P. and Verriest E.I., On the stability of coupled delay differential and continuous time difference equations, IEEE Trans. Autom. Control, 48(8), 582-590, 2003.

[16] Pepe P., On the asymptotic stability of coupled delay differential and continuous time difference equations, Automatica, 41, 107-112, 2005. 